\title{
Redirection of radio-frequency power flow by filaments
}

\author{
W. Zhang ${ }^{1}$, W. Tierens ${ }^{1}$, M. Usoltceva ${ }^{1}$, the ASDEX Upgrade team ${ }^{1}$, the EUROfusion MST1 Team ${ }^{2}$ \\ ${ }^{1}$ Max-Planck-Institut für Plasmaphysik, Garching, Germany \\ ${ }^{2}$ http://www.euro-fusionscipub.org/mst1
}

a) Corresponding author: wei.zhang@ipp.mpg.de

\begin{abstract}
When radio-frequency waves propagate through magnetized plasmas, they scatter at density filaments, which are usually aligned with the magnetic field lines. We show that this scattering can redirect part of the power from the perpendicular direction to the parallel direction (along the field lines, along the filament). In magnetic confinement fusion devices, such as tokamaks, plasma is often heated by lon Cyclotron Range of Frequency (ICRF) antennas. For such antennas, our simulations show that a parallel Poynting flux in the level of $1 \mathrm{MW} / \mathrm{m}^{2}$ can be generated by this power redirection mechanism. The increase of the magnitude of electric and magnetic fields of the wave in the filament is responsible for the parallel redirection of power flow. Simulations with constant density in the filament and background plasma show that the dominant parallel wavenumbers of the power spectrum in the filament are increased and additional wavenumbers can be generated. In the cases with an experimental density profile, only the power spectrum inside the filament is significantly affected while the rest remains almost the same.
\end{abstract}

\section{Introduction}

Radio frequency (RF) waves in the lon cyclotron range of frequencies (ICRF), electron cyclotron range of frequencies (ECRF) and lower hybrid range of frequencies (LH) are routinely used to heat magnetic confinement fusion plasmas, such as tokamaks and stellarators. The RF waves generated by antennas at the boundary of the plasma have to propagate through the turbulent scrape-off layer (SOL) before reaching the core plasma. Filaments, or blobs, are one of the main sources of SOL turbulence. They are generated near the separatrix and moves radially outward towards the wall. A filament usually has a higher density, temperature and vorticity than the background plasma, and is elongated along the magnetic field lines [1]. The higher density in the filament changes the plasma dielectric properties and thus the Stix parameters [2] which determine the wave dispersion relation. Consequently, RF waves are scattered by filaments in the SOL.

Filaments, usually with a size of a few centimeters, can have a significant influence not only on the ECRF (wavelength millimeter) and LH waves (wavelength centimeter), but also on the ICRF waves (wavelength decimeter). Previously, a lot of efforts were devoted to understand the RF wave scattering by density filaments [3-8]. Myra developed theories of scattering of ICRF fast wave and LH slow wave by a cylindrical filament [5]. 
Ram worked out the exact solution for the scattering of plane waves (ECRF and LH waves) in cold magnetized plasma at a cylindrical density filament [4]. Recently, interests are concentrating on calculating the scattering effect of EC waves by turbulence in ITER [7]. In this paper, the redirection of ICRF power flow from the radial direction to the parallel direction is studied for the first time. This mechanism will be tested on various cases, including one with an experimental density profile.

It is known that the ICRF antennas have long suffered from "hot spots", parts of the antenna that become hotter than expected $[9,10]$, and limit the amount of power that the antenna can transmit. It has long been thought that the root cause of this phenomenon is sheath rectification [11], which resulted in attempts to suppress it by minimizing the electric field component parallel to the confining magnetic field. The redirection of RF power by density filaments in the SOL, as will be studied in this paper, might be one of the sources that reinforce any power loss mechanism already present without filament, for instance the resistive losses described in section 4.

The rest of the paper is organized as follows. In section 2, a 3D COMSOL model with simplified antenna geometry is developed. Settings of the plasma and antenna parameters are explained. In section 3, particular plasma scenarios with constant density in the filament and background are studied. In section 4, an experimental density profile and a filament of typical size and density are used in the simulation. Summary and conclusions are given in section 5.

\section{Simulation setups}

\subsection{Simulation model}

A 3D COMSOL [12] model is developed for the study of power redirection in the paper. It makes use of the RAPLICASOL code $[13,14]$ and solves the same equations. The $(x, y, z)$ coordinate in the model represents the (radial, poloidal, toroidal) coordinate in a tokamak. A simplified ASDEX Upgrade (AUG) 2-strap antenna is built (Fig. 1). The spatial parameters of the antenna correspond to the real antenna: strap width of $0.2 \mathrm{~m}$, limiter width of $0.1 \mathrm{~m}$, strap-strap distance of $0.2 \mathrm{~m}$ and strap-limiter distance of $0.1 \mathrm{~m}$ in the toroidal direction; a distance of $0.03 \mathrm{~m}$ between the leading edge of limiter and strap in the radial direction. The straps are assumed to be flat, and there is no Faraday screen. The main reason of simplifying the antenna geometry is to save computational memory, so that more memory can be used to solve complex physics in the plasma region, especially when the plasma domain has a large radial and toroidal extent and the regions near the filament need to be well resolved. Despite the simplification, this 2-strap antenna still excites the same power spectrum as the real antenna [15]. For instance, the dominant parallel wavenumber in the power spectrum is $k_{z}=8 \mathrm{~m}^{-1}$, which is true in reality.

The waves are excited by setting currents on the antenna straps. Currents of $1 \mathrm{kA} / \mathrm{m}^{2}$, which are of the same level as in experiments, are set to flow in the positive and negative y directions on the two straps, respectively. Consequently, a dipole antenna phasing is produced. The wave field is calculated from the wave equation that combines the Faraday's and Ampere's equations, in the following form: 


$$
\nabla \times \mu_{r}^{-1}(\nabla \times E)-k_{0}^{2} \epsilon_{r} E=0
$$

in which $\epsilon_{r}$ is the cold plasma dielectric tensor and $\mu_{r}$ is the relative permeability. Here, we use a typical ICRF frequency of $\mathrm{f}_{\mathrm{ICRF}}=36.5 \mathrm{MHz}$ and background magnetic field of $\mathrm{B}_{0}=2 \mathrm{~T}$ in the $\mathrm{z}$ direction. The simulated plasma region is surrounded everywhere by Perfect Matching Layers (PMLs) except the $y$-z boundary on the antenna side $(x=0.2 \mathrm{~m})$. On this surface, a metallic wall boundary condition is defined, so that the waves are reflected there, as in reality.

A cold plasma assumption ( $\mathrm{Te} \sim 5 \mathrm{eV}$ ) is used in the model, thus no collisionless/Landau absorption by the plasma is considered. In addition, the collision losses are also not considered in the simulations. The dimensions of the simulated plasma in the $(x, y, z)$ direction are $(0.4 \mathrm{~m}, 0.2 \mathrm{~m}, 1.4 \mathrm{~m})$, respectively, so that the simulation region is large enough to take into account the wave scattering by filaments. The simulated plasma region is divided into the filament and the background plasma. The cylindrical filament, with its center at $\left(x_{0}, y_{0}\right)=$ $(0.12 \mathrm{~m}, 0.0 \mathrm{~m})$, is parallel to the magnetic field lines, i.e. in the $z$ direction. The plasma is composed of $95 \% \mathrm{D}$ and $5 \% \mathrm{H}$, as in typical ICRH minority heating experiments. The grid resolution in and near the filament (mesh size in the level of $3 \times 3 \times 3 \mathrm{~mm}^{3}$ ) is considerably higher than the grid resolution in the background plasma (mesh size in the level of $12 \times 12 \times 12 \mathrm{~mm}^{2}$ ). Since the radial wavelength of the fast wave is in the level of decimeter and the evanescence length of the slow wave is in the level of millimeter for the studied cases, the grid size necessary to properly resolve the fast wave and the slow wave are centimeter and submillimeter, respectively. With the current COMSOL grid resolution, we are however unable to resolve the slow wave properly. To make the grid fine enough to resolve the slow wave, the simulation region has to be significantly reduced due to the limitation of computation memory. Nevertheless, the slow wave can be well calculated in the analytical model.

(a) Top view

$-0.5$

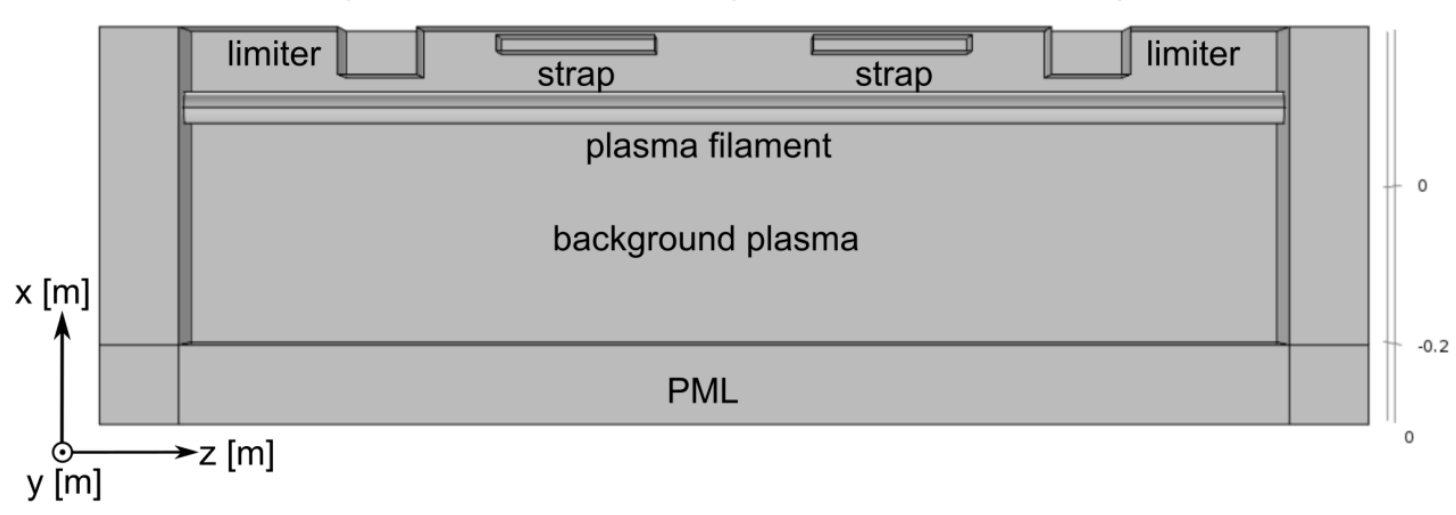

(b) Side view

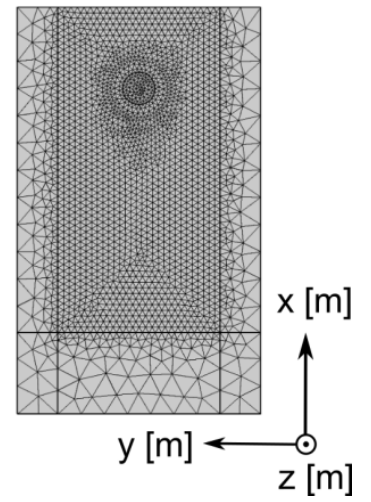

Figure 1. (a) Top and (b) side view of the 3D COMSOL model calculating the plasma-wave interaction.

\subsection{Comparison with the theory model}

Previously, Ram and Hizanidis worked out the theoretical model of the scattering of plane waves by density filaments [4]. In the model, a cylindrical coordinate system $(\rho, \phi, z)$ is used and the filament is assumed to be infinitely long and aligned with the magnetic field lines, i.e. in the $z$ direction. In the analytical "Mie" scattering, the wave fields of the incident wave, the reflected wave and the wave in the filament are calculated, in which 
the incident plane wave fields are calculated from the wave equations. The wave fields of the reflected wave are expressed as a sum over Hankel functions in cylindrical coordinates, and the wave fields of the incident wave and "internal" wave in the filament are expressed as a sum over Bessel functions in cylindrical coordinates. The amplitudes of the reflected and internal waves are calculated from the boundary conditions on the filament interface. More details of the equations are referred to reference [4]. From these equations, the magnetic field and thus the Poynting flux in the filament can be calculated.

A 2D COMSOL model is built and compared with the theoretical model. All settings in the 2D COMSOL model, including the grid resolution in the filament ( $3 \mathrm{~mm}$ ) and background plasma ( $12 \mathrm{~mm})$, are the same as the 3D one mentioned above. In the 2D model, the waves are excited by a line current at the left boundary of the simulation domain, and all other boundaries use PMLs. The following plasma and wave parameters are assumed to be the same in both the 2D COMSOL and theoretical models: background plasma density $n_{e, 0}=5 \mathrm{e} 19 \mathrm{~m}^{-3}$, filament density $n_{e, F}=1 \mathrm{e} 20 \mathrm{~m}^{-3}$, radius of the filament $\mathrm{r}_{\mathrm{F}}=0.02 \mathrm{~m}$, background magnetic field $B_{0}=2.0 \mathrm{~T}$ (in the $z$ direction), wave frequency $f_{0}=36.5 \mathrm{e} 6 \mathrm{MHz}$, and wavenumber in the $\mathrm{z}$ direction $k_{z}=9 \mathrm{~m}^{-1}$. These parameters are similar to those in AUG. The simulation region in the COMSOL model is rectangular while the one in the analytical model is circular. The comparison (Fig. 2) is made along the $x$-axis in the midplane ( $y=0 \mathrm{~m})$. The results show that the COMSOL model is in very good agreement with the theoretical model, though the grid resolution is not high enough to resolve the slow wave properly. As a result, the time averaged Poynting flux inside the filament is also significantly perturbed. Consequently, part of the power flow originally in the direction perpendicular to the magnetic field lines is redirected into the parallel direction. The averaged Poynting flux inside the filament is about five times larger than the case without filament.

Although the theoretical calculations necessarily assume uniform density in both the filament and the background, in COMSOL calculations we are able to relax this assumption and use density profiles both in the background and filaments. 

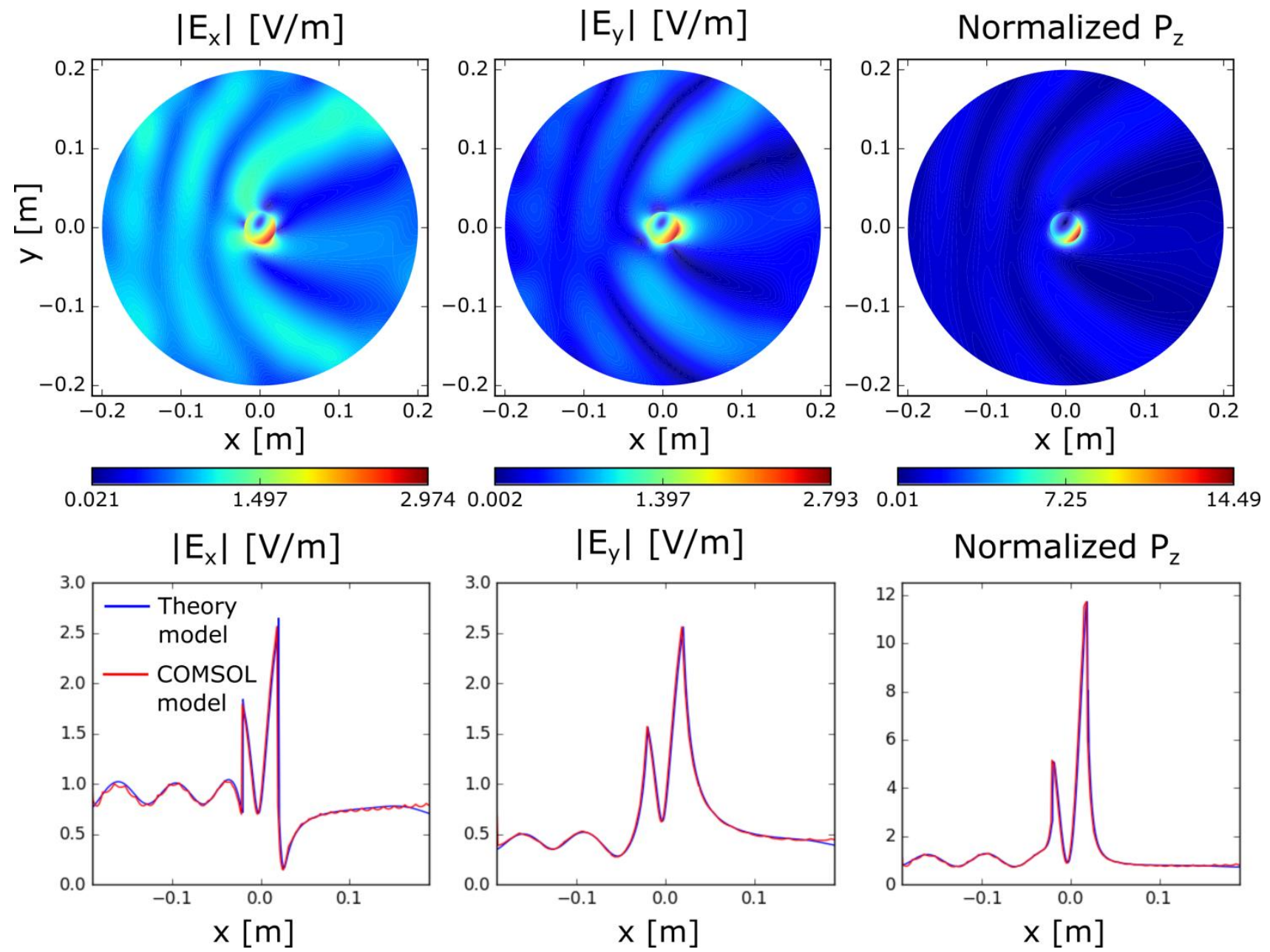

Figure 2: Top row: $\left|E_{x}\right|,\left|E_{y}\right|$ and the normalized power flow $P_{z}$ (normalized to the case without filament) calculated with the theoretical model. The filament sits at the center of the simulation region and has a radius of $0.02 \mathrm{~m}$. The wave propagates from the left to the right. Bottom row: comparisons between the theoretical model and the COMSOL model for $\left|E_{x}\right|,\left|E_{y}\right|$ and $P_{z}$ at $y=0 \mathrm{~m}$.

\subsection{Cut-off density}

Different plasma density leads to different wave propagation. It is important to understand whether the wave is propagating or evanescent for a constant density in the filament or background plasma. For this, the dispersion equation and the cut-off densities of the fast wave and slow wave are calculated [16]. For a typical parallel wavenumber $\mathrm{k}_{\mathrm{z}}=8 \mathrm{~m}^{-1}$, magnetic field $\mathrm{B}_{0}=2 \mathrm{~T}$ and wave frequency $\mathrm{f}_{\mathrm{ICRF}}=36.5 \mathrm{MHz}$, the dispersion relation (Fig. 3) shows that the fast wave is propagative at a density $n_{e} \geq 4 \mathrm{e} 18 \mathrm{~m}^{-3}$ and the slow wave is propagative in the density range of $\left[1.66 \mathrm{e} 13,4.16 \mathrm{e} 16 \mathrm{~m}^{-3}\right]$. In the next section, we will use a density larger than $4 \mathrm{e} 18 \mathrm{~m}^{-3}$ for a propagating fast wave, and use a density in the range [4.16e16, 4e18 $\mathrm{m}^{-3}$ ] for an evanescent fast wave. 


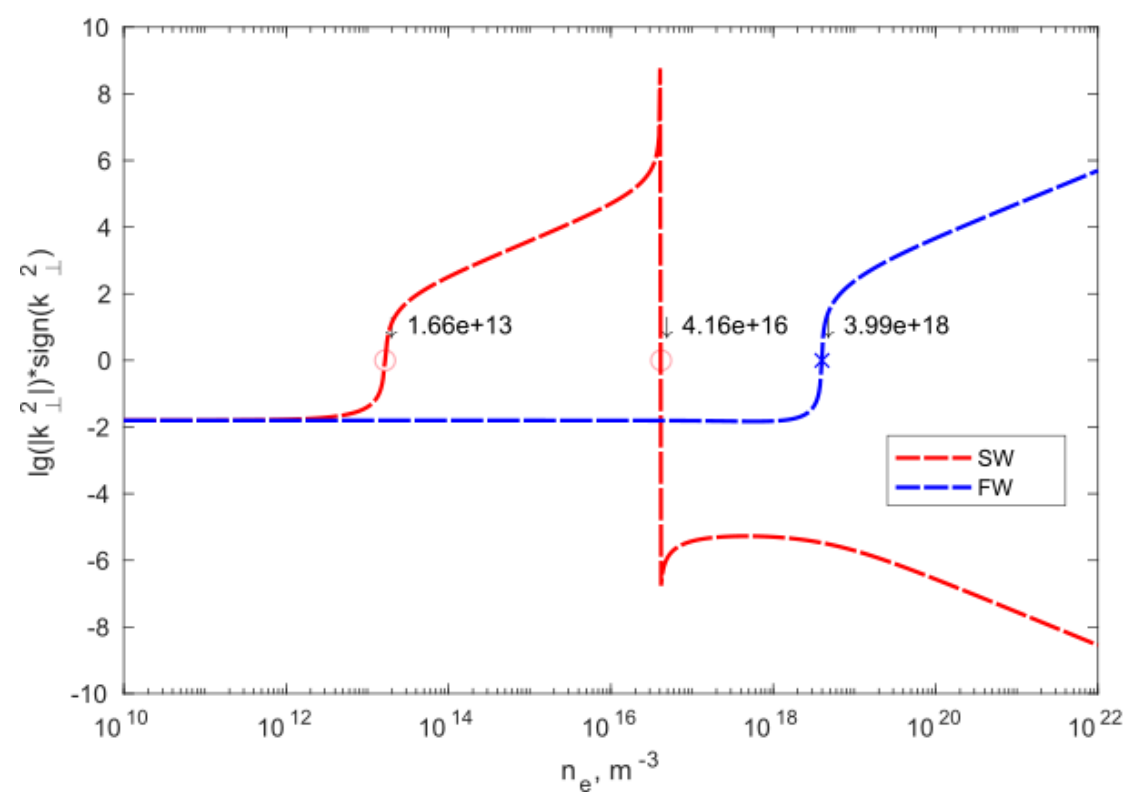

Figure 3. The dispersion relation solution of the fast wave and the slow wave, with densities at the cut-offs and at a resonance indicated. The plasma composition is $95 \% \mathrm{D}+5 \% \mathrm{H}$, ICRF frequency $\mathrm{f}_{\mathrm{ICRF}}=36.5 \mathrm{MHz}$, magnetic field $\mathrm{B}_{0}=2 \mathrm{~T}$ and parallel wavenumber $\mathrm{k}_{\mathrm{z}}=8 \mathrm{~m}^{-1}$.

\section{Simulations with constant density profiles}

\subsection{Representative scenarios}

In this section, several particular plasma scenarios with different constant densities in the filament and background are studied to examine the mechanism of RF power redirection. The studied cases are: (1) fast waves are propagative both in the background plasma and filament, with $\mathrm{n}_{\mathrm{e}, \mathrm{B}}=1 \mathrm{e} 19 \mathrm{~m}^{-3}$ and $\mathrm{n}_{\mathrm{e}, \mathrm{F}}=5 \mathrm{e} 19 \mathrm{~m}^{-3} ;(2)$ fast wave is evanescent in the background plasma and propagative in the filament, with $\mathrm{n}_{\mathrm{e}, \mathrm{B}}=5 \mathrm{e} 17 \mathrm{~m}^{-3}$ and $\mathrm{n}_{\mathrm{e}, \mathrm{F}}=5 \mathrm{e} 18 \mathrm{~m}^{-3}$; (3) fast wave is propagative in the background plasma and evanescent in the filament, with $\mathrm{n}_{\mathrm{e}, \mathrm{B}}=5 \mathrm{e} 19 \mathrm{~m}^{-3}$ and $\mathrm{n}_{\mathrm{e}, \mathrm{F}}=5 \mathrm{e} 17 \mathrm{~m}^{-3}$. Except the scenario which the fast wave is both evanescent in the background and filament, the studied cases are representative of all scenarios. Scenario (1) is very common while scenario (2) and scenario (3) may not often been seen in fusion plasmas. Moreover, cases where only the slow wave becomes resonant are avoided in the present paper. Since the typical speed of the ICRF fast waves (in the level of $10^{6} \mathrm{~m} / \mathrm{s}$ ) is much faster than that of filaments (in the level of $10^{3} \mathrm{~m} / \mathrm{s}$ ), the filament is considered as immobile in the simulations. The presented results are in steady-states since frequency domain is considered.

For the studied cases, we will show the perturbed wave fields, Poynting flux and the Energy Spectrum Density (ESD). The 2D ESD is generated by 1D Fourier transform, in the parallel direction, of the $2 \mathrm{D}$ electric field $\mathrm{E}_{\mathrm{x}}$ in a radial-parallel plane through the filament. It is calculated by $\operatorname{ESD}\left(x, k_{\|}\right)=\left|\widehat{E}\left(x, k_{\|}\right)\right|^{2}$, where $\hat{E}\left(x, k_{\|}\right)=$ $\int_{z_{\text {left }}}^{z_{\text {right }}} E_{x}(x, z) \exp \left(-i k_{\|} z\right) d z$ using Fast Fourier Transform along the $z$ coordinate. Here, $z_{\text {right }}$ and $z_{\text {left }}$ are 
the $z$ coordinates at the right and left boundary of the simulation domain, respectively. $k_{\|}$is the parallel wavenumber.

The calculated results for these cases are shown in Fig. 4. In case (1), the electric field in the filament is increased almost by three times while the wavelength in the filament reduces to one third as before. A strong reflection of RF power happens radially at the filament location because of the very high density in the filament. The perturbation of wave fields and thus the redirection of RF power in the filament are very significant. For instance, $\mathrm{P}_{\mathrm{z}}$ can be as large as $10 \mathrm{MW} / \mathrm{m}^{2}$ and almost does not decay toroidally. The dominant wavenumber is changed from $k_{\|}=7 m^{-1}$ to $k_{\|}=11 m^{-1}$, accompanied with the generation of secondary wavenumbers $k_{\|}=16$ $m^{-1}$ and $k_{\|}=22 m^{-1}$.

In case (2), the fast wave is only propagative in the filament and is evanescent in the background plasma. The fast wave has to tunnel through the evanescent layer before reaching the plasma. Upon reaching the filament, the wave propagates along the filament, and the effect of the RF power redirection in the parallel direction is very significant. The wave fields, the Poynting flux and the power spectrum are only significant in the filament. The redirected power flow, which is in the level of $0.3 \mathrm{MW} / \mathrm{m}^{2}$, almost does not decay in the parallel direction. Two wavenumbers, the dominant one $k_{\|}=10 \mathrm{~m}^{-1}$ and the secondary one $k_{\|}=5 \mathrm{~m}^{-1}$, are generated in the filament. In the periphery of the filament, the ESD decays rapidly and then disappears. In addition, case (2) with radial PML closer to the antenna and higher filament density (background density $1 \mathrm{e} 17 \mathrm{~m}^{-3}$ ) shows that the magnitude of the power redirection is reduced, but this effect persists.

In case (3), the fast wave is propagative in the background plasma and evanescent in the filament. The wave fields, the Poynting flux and the power spectrum are radially discontinuous on the filament boundaries. The redirection of RF power in the parallel direction is also obvious. A large amount of power is redirected along the filament surfaces as the fast wave is evanescent in the filament. The dominant wavenumber $k_{\|}=8 \mathrm{~m}^{-1}$ disappears in the filament. In contrast, the secondary wavenumber $k_{\|}=20 \mathrm{~m}^{-1}$ survives in the filament, but soon disappears after passing through the filament. Beyond the filament, the dominant wavenumber come into being again.

Although the slow wave cannot be properly resolved in the COMSOL model, it can be well solved in the analytical model. Based on an analytic solution of the wave equation [4], we can calculate the contribution of the electric field of the fast wave and slow wave separately. The results (Fig. 5) show that both the fast and slow wave electric field peaks at a certain $\mathrm{k}_{\|}$value in each case. Although in this calculation it does not take into account the antenna spectrum used in the simulations and only uses plane waves, it still roughly predicts the peak $\mathrm{k}_{\|}$in the filaments in figure 4 . For the wave components with these new $\mathrm{k}_{\|}$peaks in the spectrum, the wave is localized in the filament in the cross-section perpendicular to the magnetic field lines, but propagates along the filament without decaying. 
(1) $\mathrm{n}_{\mathrm{e}, \mathrm{B}}=1 \mathrm{e} 19 \mathrm{~m}^{-3}$, fast wave propagative

$\mathrm{n}_{\mathrm{e}, \mathrm{F}}=5 \mathrm{e} 19 \mathrm{~m}^{-3}$, fast wave propagative
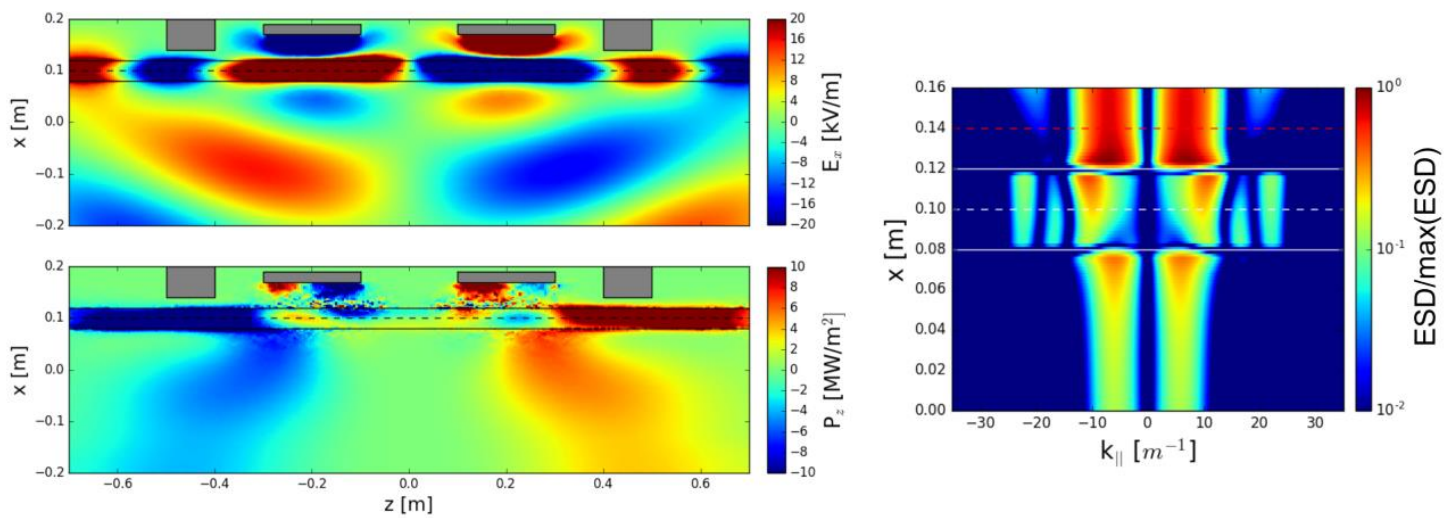

(2) $\begin{aligned} & \mathrm{n}_{\mathrm{e}, \mathrm{B}}=5 \mathrm{e} 17 \mathrm{~m}^{-3} \text {, fast wave evanescent } \\ & \mathrm{n}_{\mathrm{e}, \mathrm{F}}=5 \mathrm{e} 18 \mathrm{~m}^{-3} \text {, fast wave propagative }\end{aligned}$
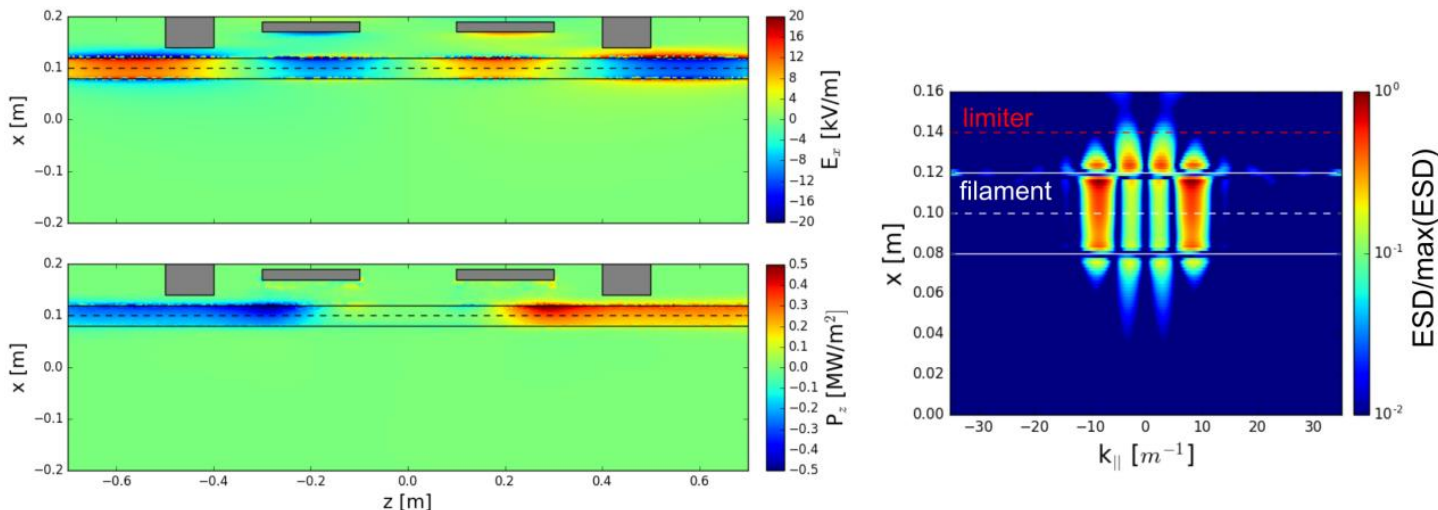

(3) $\mathrm{n}_{\mathrm{e}, \mathrm{B}}=5 \mathrm{e} 19 \mathrm{~m}^{-3}$, fast wave propagative $\mathrm{n}_{\mathrm{e}, \mathrm{F}}=5 \mathrm{e} 17 \mathrm{~m}^{-3}$, fast wave evanescent
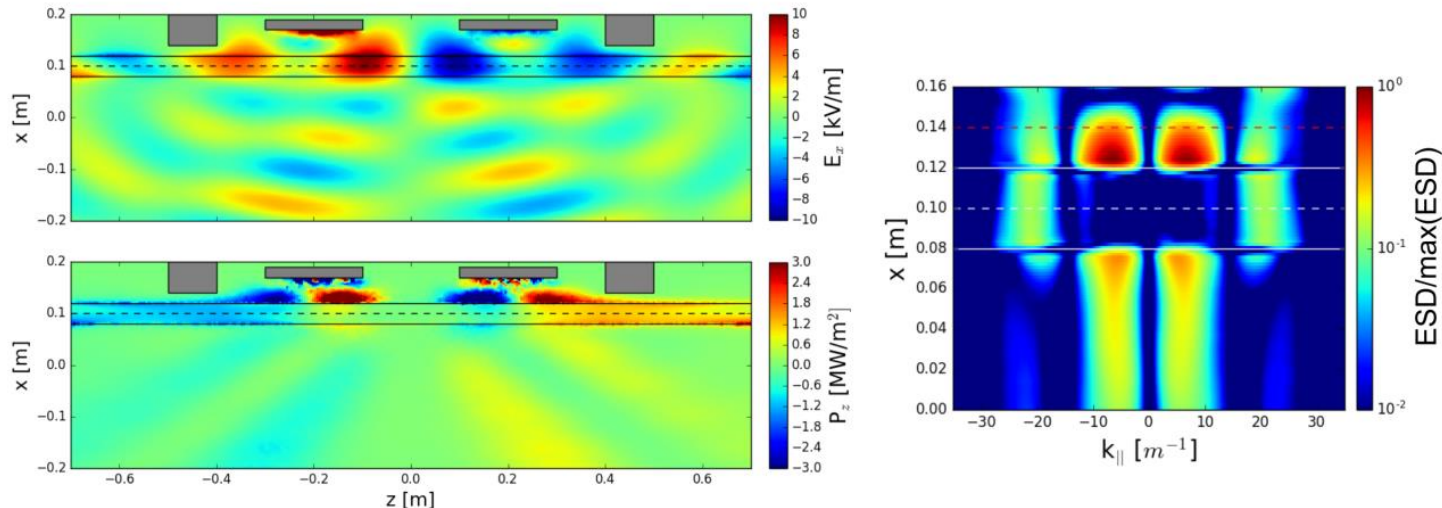

Figure 4. The calculated $x$ component of electric field, Poynting flux and the Energy Spectrum Density (ESD) for the three particular cases. The white lines depict the boundaries of the filament and the red dashed line is the location of the leading edge of limiter. 

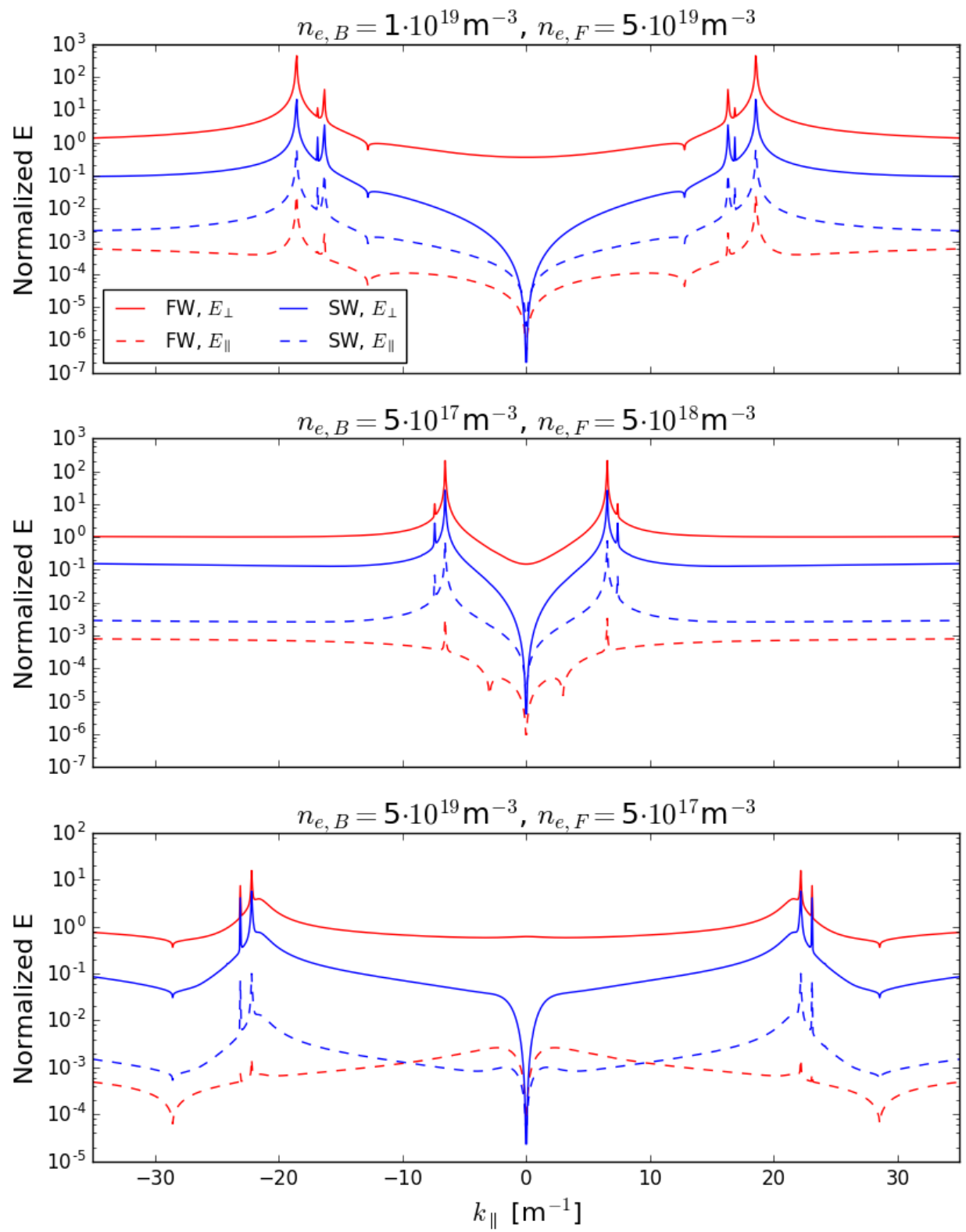

Figure 5. Analytical calculations of the normalized perpendicular and parallel electric fields for the fast wave (FW) and slow wave (SW), respectively. All electric fields are normalized to the incident fast wave $E_{\perp}$ amplitude.

\subsection{Grid resolution}

In the 3D COMSOL model, the grid resolution in the filament $(\sim 3 \mathrm{~mm})$ and background plasma $(\sim 12 \mathrm{~mm})$ is not fine enough to resolve the slow wave. To check the accuracy of the results, the same grid resolution is used in the 2D COMSOL model to perform simulations which can then be compared with the analytical ones. All plasma and wave parameters are set as the same in two models. The comparisons are made in the midplane $(\mathrm{y}=0.0 \mathrm{~m})$ as in the previous section. The results (Fig. 6) show that in case (1) and case (3), all wave fields calculated by the 2D COMSOL model have good agreement with the analytical ones. However, in case (2), the wave fields and the 
normalized power flow in a small region near the boundary of the filament are underestimated by the 2D COMSOL model. This is because at the boundary of the filament, intensive mode conversion of fast wave to slow wave happens. Due to the limitation of the grid resolution, the wave fields of the slow wave are not well resolved. Nevertheless, the simulation results in all other regions still show good agreement with the analytical ones.

(1) $n_{e, B}=1 e 19 m^{-3}, n_{e, F}=5 e 19 m^{-3}$
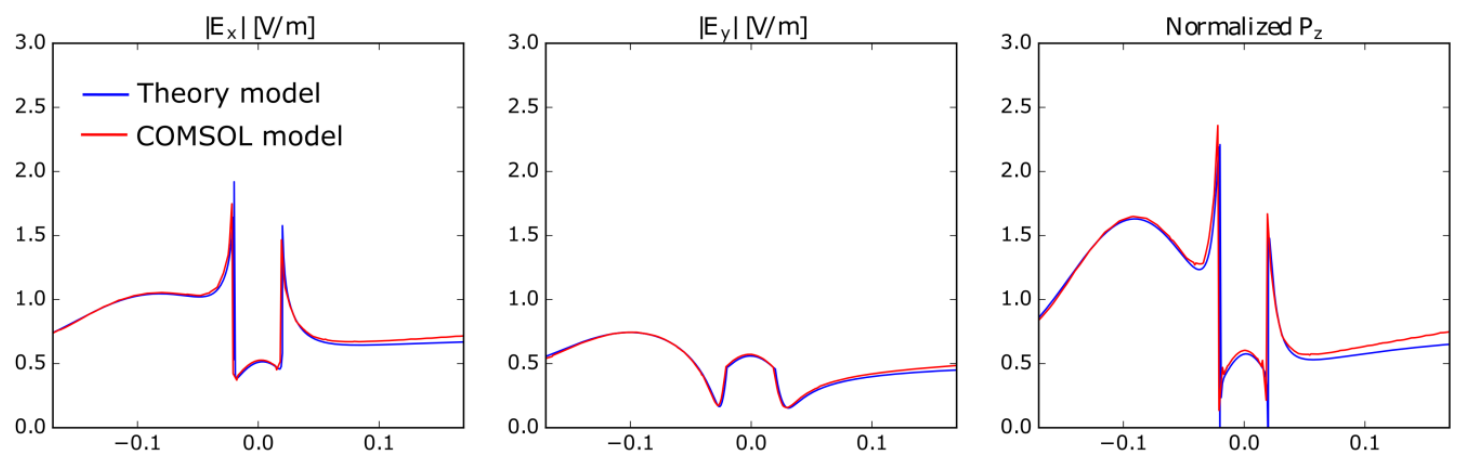

(2) $n_{e, B}=5 e 17 m^{-3}, n_{e, F}=5 e 18 m^{-3}$
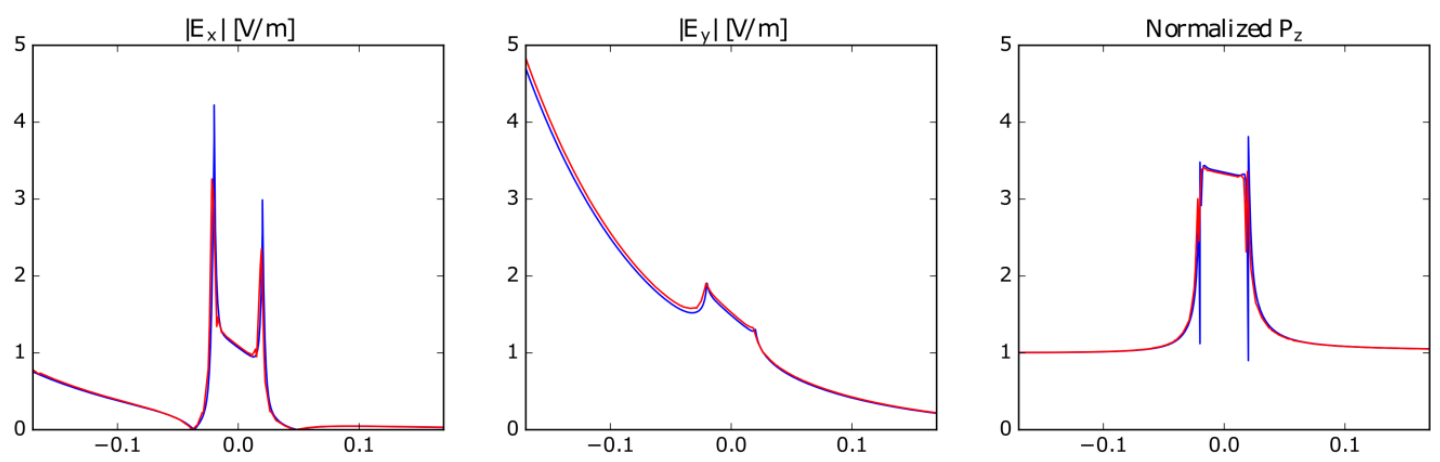

(3) $n_{e, B}=5 e 19 m^{-3}, n_{e, F}=5 e 17 m^{-3}$
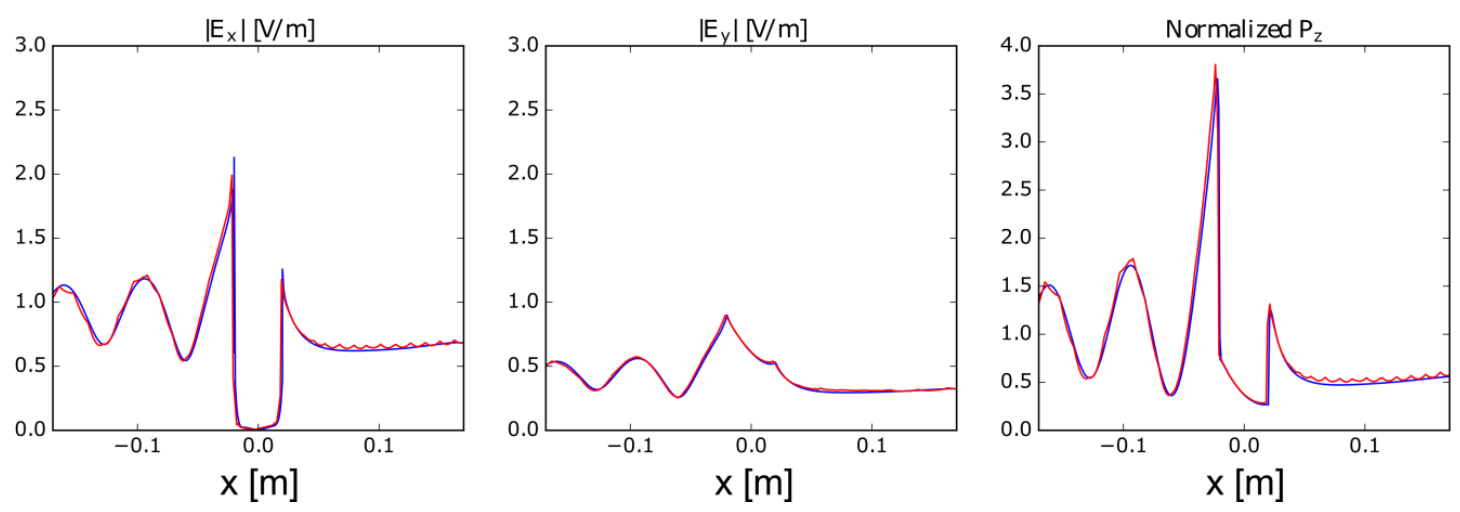

Figure 6. Comparisons of $\left|E_{x}\right|,\left|E_{y}\right|$ and the normalized power flow $P_{z}$ (normalized to the case without filament) calculated with the analytical model and the 2D COMSOL mode. In both models, the parallel wave vector is set as $k_{z}=9 \mathrm{~m}^{-1}$ and the filament radius is $2 \mathrm{~cm}$. 


\subsection{Boundary conditions}

In a tokamak, the magnetic field lines in the SOL are terminated by the divertor plates or the first wall. We assume that the filaments are located in the SOL in our simulations. Since the connection length of the magnetic field lines in the SOL of AUG is $\sim 60 \mathrm{~m}$. For such a long connection length, it is possible that the wave can be dissipated through collision losses in its way to the divertor. For those waves which are able to propagate far enough along the filament to reach the divertor, a far-field rectified sheath can be generated [17]. It is thought as a possible RF power loss mechanism in the SOL [18]. Moreover, it is far from obvious that, when they reflect from a sheath boundary condition, that the reflected wave is again able to propagate along the filament back to the antenna. If they don't, the power leaks into the electric fields near the divertor and doesn't make it back to the antenna, even if it is not immediately absorbed. Based on these reasons, we consider it is reasonable to use parallel PMLs as boundary conditions.

Simulations are done with different toroidal extensions of the simulation region and different boundary conditions (Fig. 7). It is shown that the redirected power remains roughly the same when the toroidal extension of the simulation region is increased. The redirected parallel power, however, decreases significantly when the PMLs are changed to toroidal periodic boundary conditions. This is because when assuming a periodic boundary condition, the $P_{\mathrm{z}}$ values at the two boundaries are forced to be the same. As a result, $P_{\mathrm{z}}$ becomes zero at both boundaries, and the power redirection phenomenon is dramatically reduced. However, using a toroidal periodic boundary condition essentially assumes that the field lines are connected to themselves at the two ends, which is not true both in our simulations and in reality.

The simulations with toroidal PMLs feature RF fields that may be different at the two toroidal extremities of the main simulation domain. Fourier theory treats such RF fields as toroidally periodic with a sharp discontinuity at the toroidal borders, which impacts the Poynting vectors. For example in the initial maps $\mathrm{P}_{\mathrm{z}}$ exhibits opposite values at the two opposite toroidal sides, while for any periodic field $P_{z}$ is the same at the two toroidal sides. $P_{x}$ and $\mathrm{P}_{\mathrm{z}}$ are related by the energy conservation equation $\nabla \cdot \overrightarrow{\mathrm{P}}=0$ so all components of the Poynting vector may be affected. Such artefacts are probably insignificant in most cases, e.g. in the simulations with inhomogeneous density in section 4, because RF field intensities are small at the toroidal boundaries, compared to their maximal values. But in extreme situations like case 2 , it is shown numerically that replacing the PMLs by periodic boundary conditions can have macroscopic effects on the Poynting vectors over the whole simulation domain. 


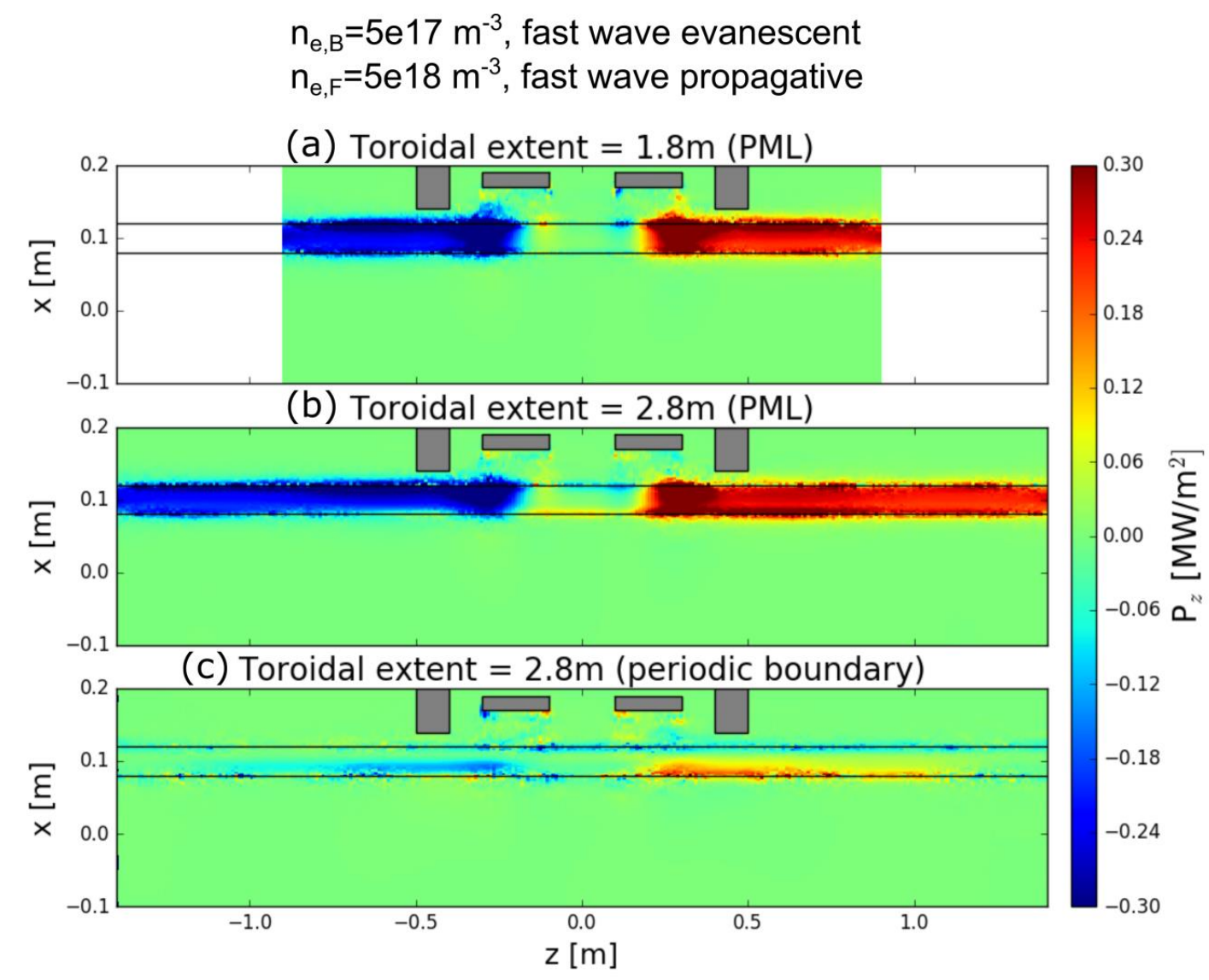

Figure 7. Simulations with different parallel extensions of the simulation domain and boundary conditions for scenario (2) with background density $5 \mathrm{e} 17 \mathrm{~m}^{-1}$ and filament density $5 \mathrm{e} 18 \mathrm{~m}^{-1}$. (a) Parallel extension is $1.8 \mathrm{~m}$ and PML boundaries; (b) Parallel extension is $2.8 \mathrm{~m}$ and PML boundaries; (c) parallel extension is $2.8 \mathrm{~m}$ and toroidal periodic boundaries.

\subsection{Parameter scan}

To have a more quantitative understanding of the parallel power flow redirection, parameter scans are performed. Given a specific background plasma density and RF wave frequency, the amount of RF wave power coupled to the parallel direction depends on the radius of the filament, $\mathrm{r}_{\mathrm{F}}$, and the density ratio between the filament and background plasma, $\mathrm{n}_{\mathrm{e}, \mathrm{F}} / \mathrm{n}_{\mathrm{e}, \mathrm{B}}$. Here, the 3D averaged parallel Poynting flux over the filament volume $\left\langle\left|P_{Z}\right|\right\rangle$ is calculated. The results (Fig. 8) indicate that $\left\langle\left|P_{z}\right|\right\rangle$ increases nonlinearly as the density ratio increases. For a fixed $\mathrm{r}_{\mathrm{F}}=2 \mathrm{~cm},\left\langle\left|P_{\mathrm{Z}}\right|\right\rangle$ first increases only in a small level when the density ratio $\mathrm{n}_{\mathrm{e}, \mathrm{F}} / \mathrm{n}_{\mathrm{e}, \mathrm{B}}$ is lower than 2. This increase becomes significantly larger once $\mathrm{n}_{\mathrm{e}, \mathrm{F}} / \mathrm{n}_{\mathrm{e}, \mathrm{B}}$ exceeds 2 . For a fixed $\mathrm{n}_{\mathrm{e}, \mathrm{F}} / \mathrm{n}_{\mathrm{e}, \mathrm{B}}$, the change of $\left\langle\left|P_{z}\right|\right\rangle$ is similar to a parabola as the filament radius increases, and it peaks when the filament radius is close to $1.75 \mathrm{~cm}$. The filament radius which generates the peak value may change as the radial wavelength of fast wave varies. 

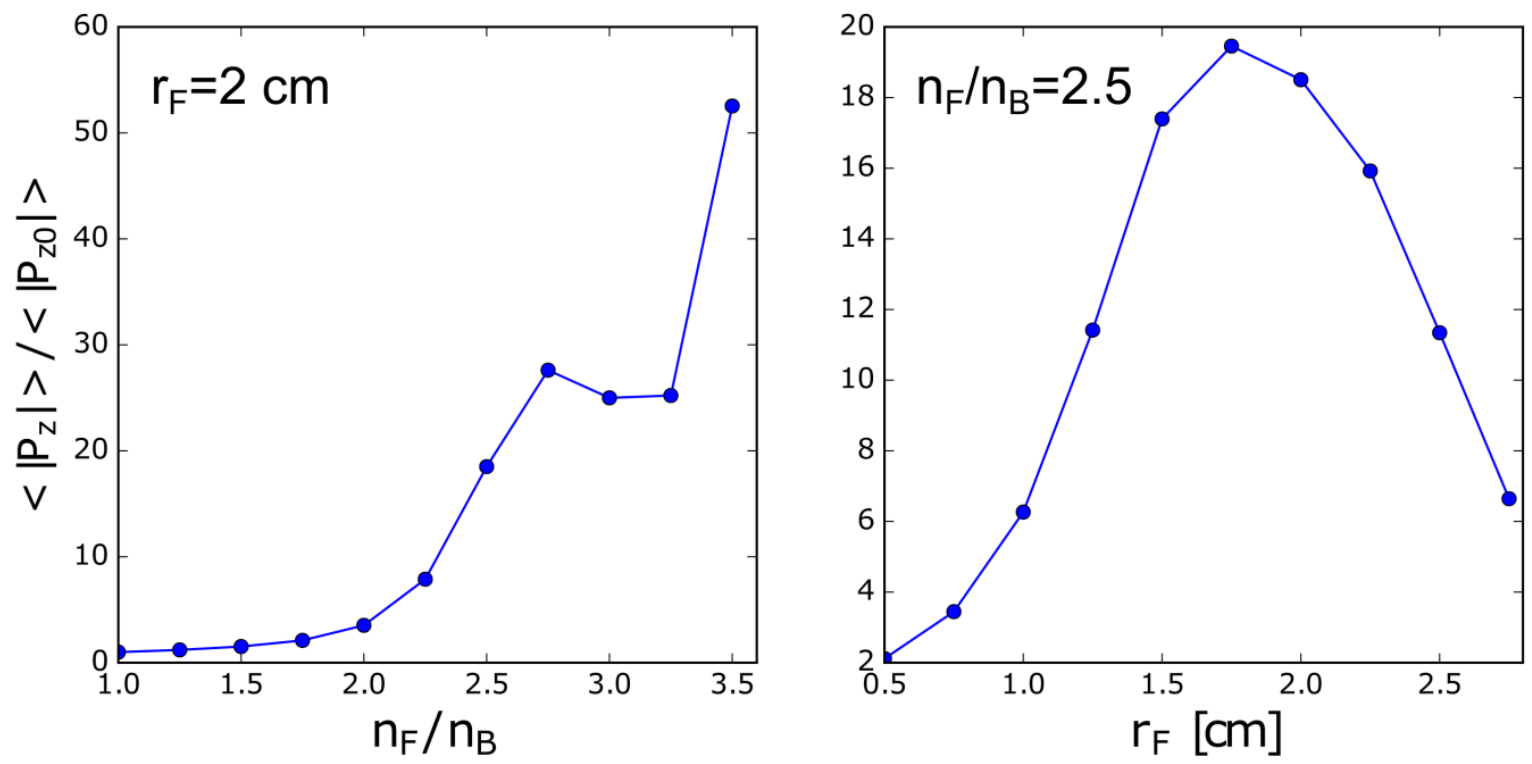

Figure 8. Averaged parallel Poynting flux in the filament $\left(\left\langle\left|P_{z}\right|\right\rangle\right)$ as a function of (a) filament density and (b) filament radius. The density in the background is equal to $1 \mathrm{e} 19 \mathrm{~m}^{-3}$. The averaged parallel Poynting flux is normalized to the value without filament $\left(\left\langle\left|P_{z 0}\right|\right\rangle\right)$.

\section{Simulations with experimental profiles}

\subsection{Experimental density profile}

In this section, an experimental density profile of a typical H-mode (\#31269, 2.1s) is used (Fig. 9). The experimental density profile is adjusted to the $x$ coordinate with the strap as a reference. From the cut-off densities of the fast wave and slow wave (Fig. 3), it can be pointed out that for the selected experimental density profile, the slow wave generated by the antenna only exists in a very thin plasma layer ( $\sim \mathrm{a}$ few $\mathrm{mm}$ ) in front of the strap. The fast wave, however, is propagative almost everywhere in the simulation region $(-0.200$ $\mathrm{m} \leq \mathrm{x} \leq 0.144 \mathrm{~m}$ ). In this section, the filament is set $0.03 \mathrm{~m}$ radially away from the strap, which is out of the propagation region of slow wave. Thus, only interaction between the filament and the fast wave is considered.

In AUG, the typical density ratio between the filament and the background plasma $\left(\mathrm{n}_{\mathrm{e}, \mathrm{F}} / \mathrm{n}_{\mathrm{e}, \mathrm{B}}\right)$ is in the range of 2-3, and the typical filament radius $\left(\mathrm{r}_{\mathrm{F}}\right)$ is in the range of $5 \mathrm{~mm}-4 \mathrm{~cm}$ [19-21]. In this section, typical filament parameters of $\mathrm{n}_{\mathrm{e}, \mathrm{F}} / \mathrm{n}_{\mathrm{e}, \mathrm{B}}=2$ and $\mathrm{r}_{\mathrm{F}}=0.02 \mathrm{~m}$ are used. The filament density is also a profile. 


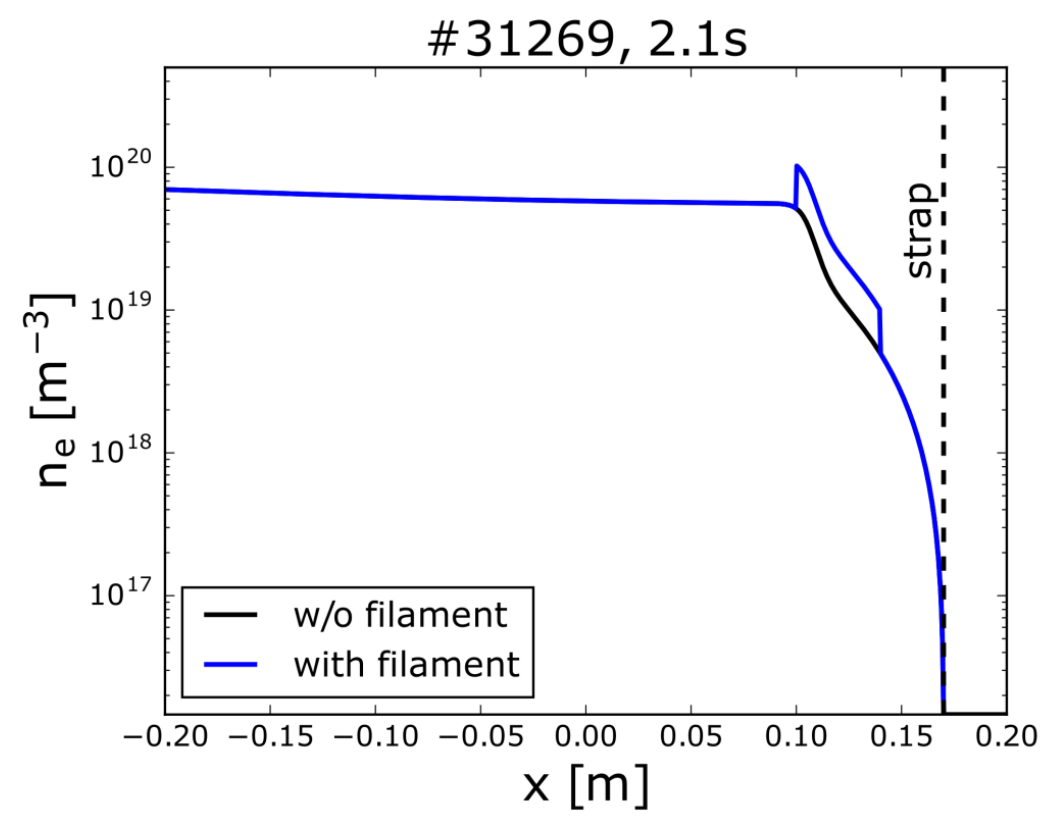

Figure 9. The experimental density profile of a standard $\mathrm{H}$-mode and the density profile with a filament used for simulations in section 3.

In reality, the antenna faces the SOL with a structure of filaments typically spaced poloidally by few centimeters $[8,22]$. In the current simulations, we use only one filament for the following reasons: firstly, the simulation region is only representative of a certain poloidal range of the antenna, and only parallel power redirection at a certain poloidal location of the antenna is studied; secondly, tests show that a local power redirection is mainly affected by a local filament.

\subsection{Perturbation of wave fields}

Due to RF wave scattering at the filament location, the wave fields in and near the filament are strongly perturbed, as shown in Fig. 10. On one hand, the magnitude of the wave fields, especially the $E_{y}$ component, is significantly increased in the filament. The averaged $E_{y}$ increases from $2.5 \mathrm{kV} / \mathrm{m}$ to $3.4 \mathrm{kV} / \mathrm{m}$ inside the filament. However, the averaged $E_{x}$ in the filament remains almost the same. On the other hand, the toroidal extent of the wave fields becomes larger in the filament. For instance, the contour of $E_{y}=2 \mathrm{kV} / \mathrm{m}$ shifts from $\mathrm{z}=0.41 \mathrm{~m}$ to $\mathrm{z}=0.53 \mathrm{~m}$ toroidally.

The perturbations of electric and magnetic fields in and near the filament subsequently lead to a perturbation of the time averaged Poynting flux, as shown in Fig. 11. The parallel power flow redirection is most prominent inside the filament since the perturbation of the wave fields is largest there. The RF power flows towards the toroidal boundaries of the simulation domain is symmetric about the center of the antenna $(\mathrm{z}=0 \mathrm{~m})$ and flows away of the antenna. For the averaged power density in the filament, $\left\langle\left|\mathrm{P}_{\mathrm{x}}\right|\right\rangle$ increases from $0.48 \mathrm{MW} / \mathrm{m}^{2}$ to 0.58 $\mathrm{MW} / \mathrm{m}^{2},\left\langle\left|\mathrm{P}_{\mathrm{y}}\right|\right\rangle$ increases from $0.40 \mathrm{MW} / \mathrm{m}^{2}$ to $0.46 \mathrm{MW} / \mathrm{m}^{2}$ and $\left\langle\left|\mathrm{P}_{\mathrm{z}}\right|\right\rangle$ increases from $0.12 \mathrm{MW} / \mathrm{m}^{2}$ to 0.38 $\mathrm{MW} / \mathrm{m}^{2}$. The increase of $\left\langle\left|\mathrm{P}_{\mathrm{y}}\right|\right\rangle$ is due to the poloidal diffraction of wave fields by the filament while the significant increase of $\left\langle\left|\mathrm{P}_{\mathrm{z}}\right|\right\rangle$ indicates that part of the RF power is redirected from the perpendicular direction to 
the parallel direction. In fact, the total power transported radially to the core, as calculated by integrating $\left|\mathrm{P}_{\mathrm{x}}\right|$ at the PML surface where the waves end $(x=-0.2 \mathrm{~m})$, decreases from $0.13 \mathrm{MW}$ to $0.11 \mathrm{MW}$. If taking into account the whole simulation region, the total power decreases from $55.0 \%$ to $51.8 \%$ in the $x$ direction, increases from $18.5 \%$ to $20.5 \%$ in the $y$ direction and increases from $26.5 \%$ to $27.7 \%$ in the $z$ direction. Most of the power leaving the simulation domain along $z$ direction through the plasma-toroidal PML boundary $(z=0.7 \mathrm{~m}$ and $-0.7 \mathrm{~m}$, $x>-0.2 \mathrm{~m})$ is lost in the radial interval $(-0.2 \mathrm{~m}<\mathrm{x}<-0.1 \mathrm{~m})$ and not in the filament region.

Besides the magnitude, the parallel/toroidal extension of $\mathrm{P}_{\mathrm{z}}$ also increases as the toroidal extension of perturbed wave fields increases. However, the magnitude of this redirected $\left|\mathrm{P}_{\mathrm{z}}\right|$ decreases quickly in the toroidal direction. For instance, it decreases from $1.0 \mathrm{MW} / \mathrm{m}^{2}$ at $(x, y, z)=(0.12,0.0,0.27 \mathrm{~m})$ to $0.07 \mathrm{MW} / \mathrm{m}^{2}$ at $(x, y, z)=(0.12,0.0,0.5 m)$. As a result, the power flow reaching the toroidal PMLs $(z= \pm 0.7 \mathrm{~m})$ barely changes.

(a) $\left|E_{x}\right|$

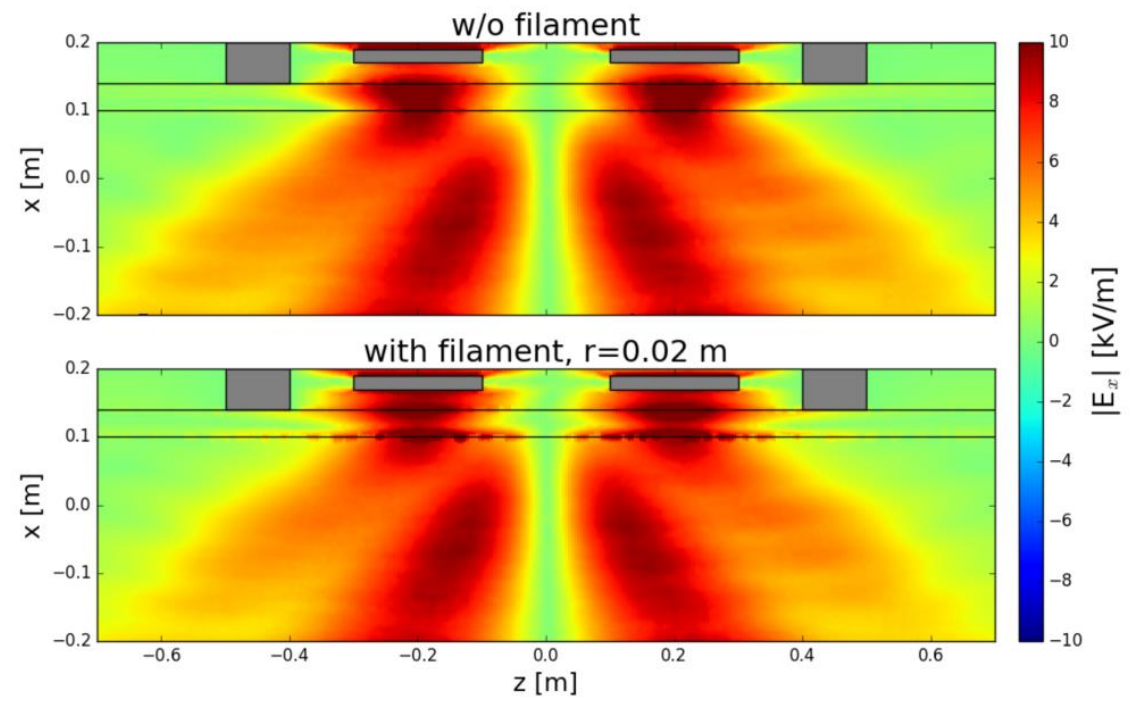

(b) $\left|E_{y}\right|$

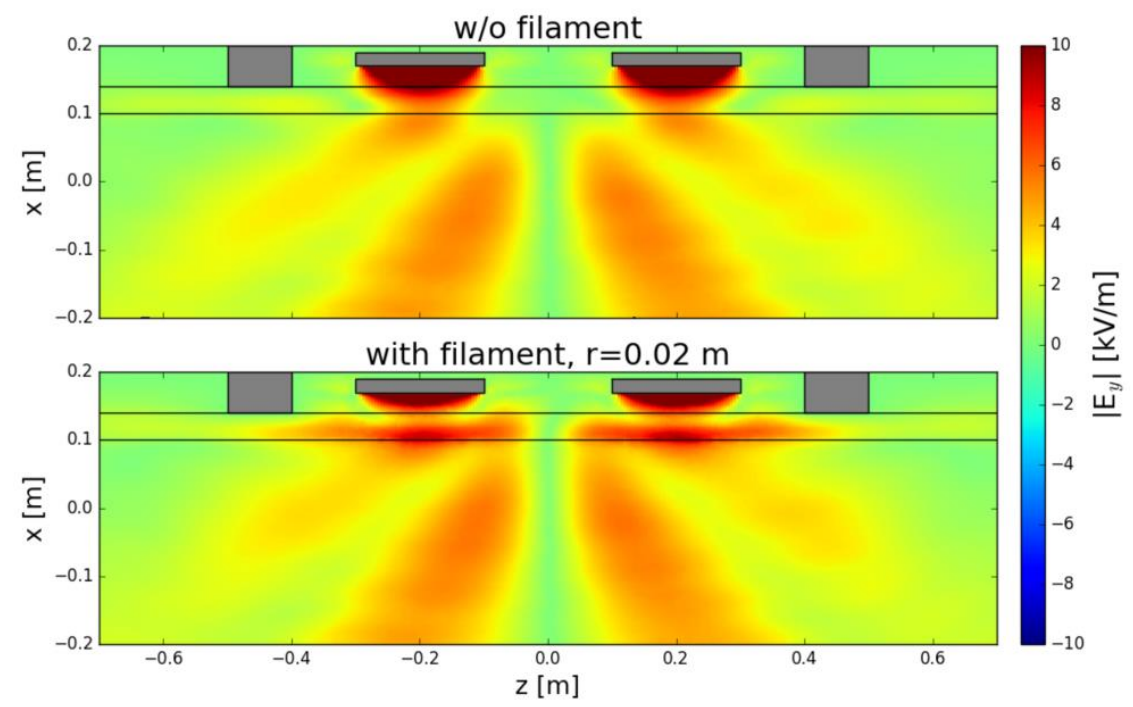


Figure 10. The $x$ and $y$ component of the electric field for the cases without and with a filament. The filament has a radius of $0.02 \mathrm{~m}$ and a density ratio of $n_{e, F} / n_{e, B}=2$. The horizontal black lines depict the locations of the filament.

(a) $P_{z}$

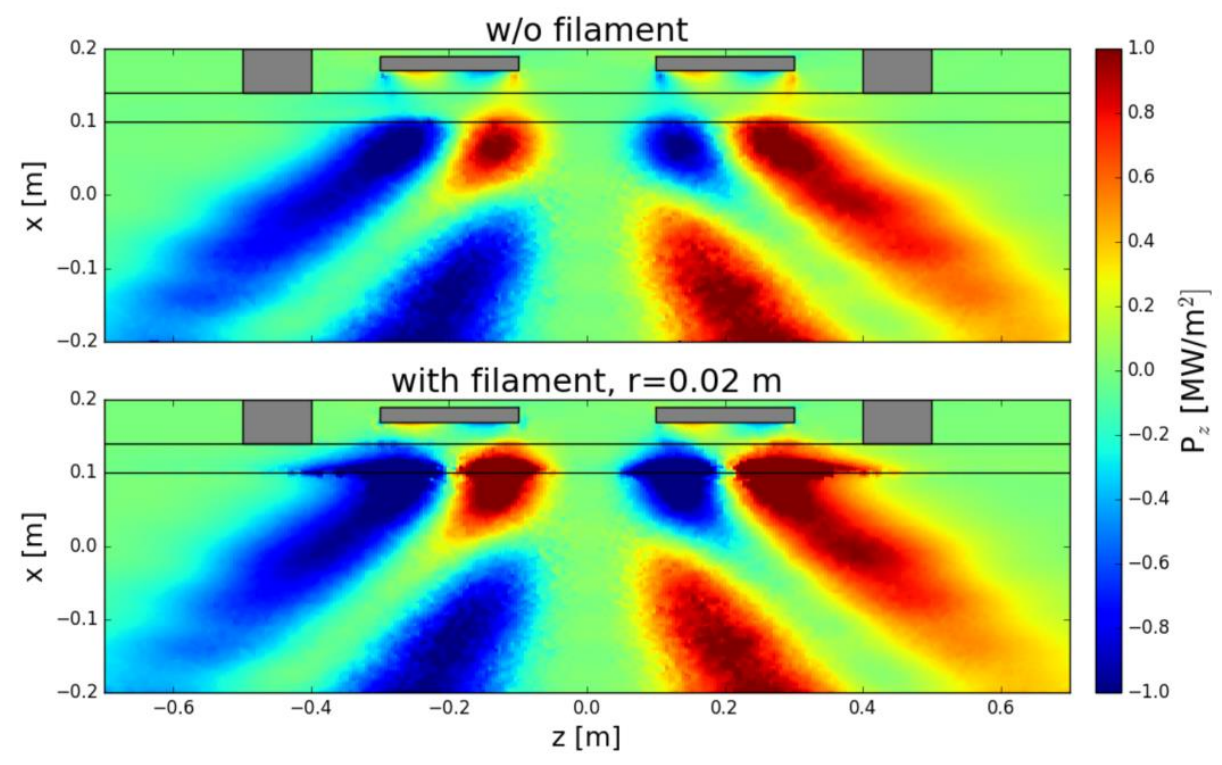

(b) $P_{x}$

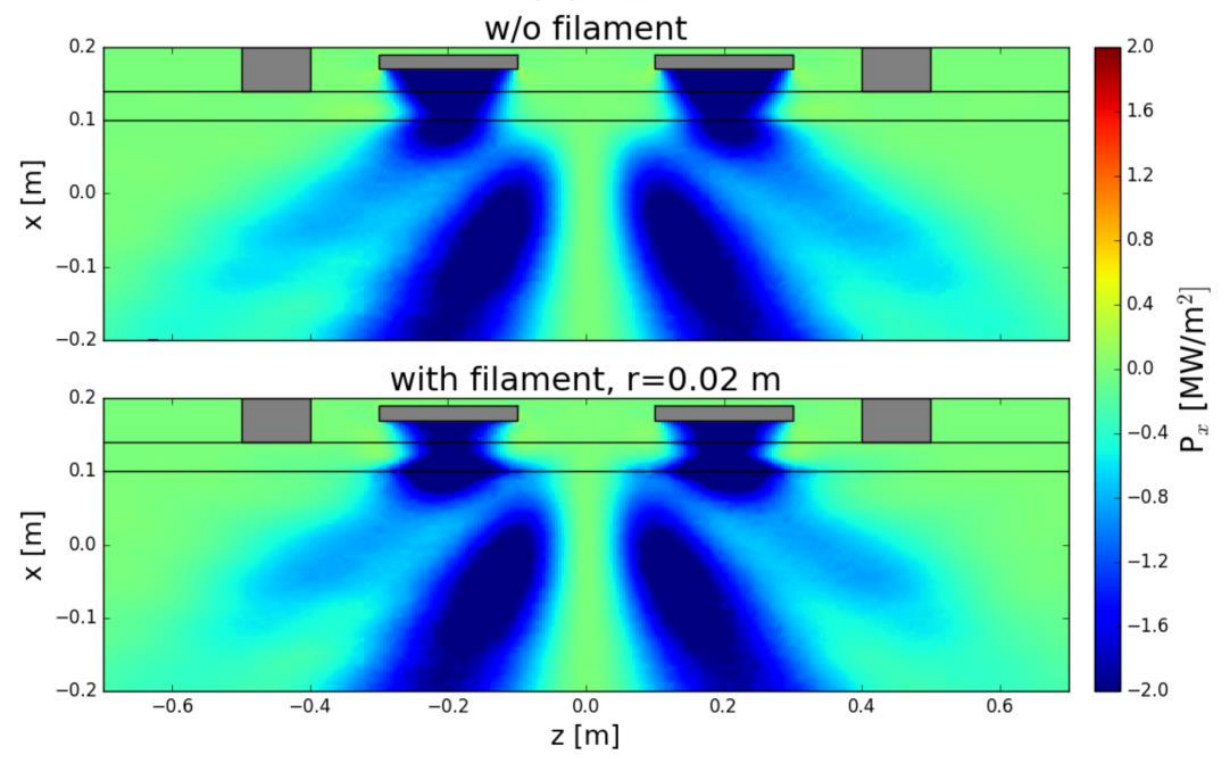

Figure 11. The $z$ and $x$ component of the time averaged Poynting flux in the $x-z$ plane $(y=0.0 \mathrm{~m})$ for the cases without and with a filament. The filament has a radius of $0.02 \mathrm{~m}$ and a density ratio of $n_{e, F} / n_{e, B}=2$. The horizontal black lines depict the locations of the filament.

Next, the power spectrum densities (ESD) for the cases without and with filament are compared. The results (Fig. 12) show that the ESD remains roughly the same near the antenna in both cases, but changes significantly in the filament. The modification of the ESD is due to the modification of the wave fields. It can be seen that the 
ESD magnitude of both the primary wavenumber $k_{\|}=8 \mathrm{~m}^{-1}$ and the secondary wavenumber $k_{\|}=21 \mathrm{~m}^{-1}$ become obviously larger in the radially outward half of the filament (i.e. $0.10 \mathrm{~m} \leq \mathrm{x} \leq 0.12 \mathrm{~m}$ ). Outside the filament region, the ESD remains almost the same.

It can be pointed out that for the case with an experimental density profile, the parallel redirection of the RF power is limited toroidally to the antenna vicinity. No new wavenumber is developed in the computed power density spectra, compared to the reference run without filament. One possible reason is that new $\mathrm{k}_{\|}$pops up if a resonance condition is fulfilled, i.e. for very specific couples of $\left(\mathrm{n}_{\mathrm{e}, \mathrm{F}}, \mathrm{n}_{\mathrm{e}, \mathrm{B}}\right)$. With inhomogeneous profiles, the resonance could be met at one radial location only, and not over the whole radial extent of the filament. The other possible reason is that we use a very large density ratio ( 10) between the filament and background plasma for the constant density cases, while in the experimental profile we only use a density ratio of 2.

\section{(a) Energy spectral density (ESD) vs. $x$}

w/o filament

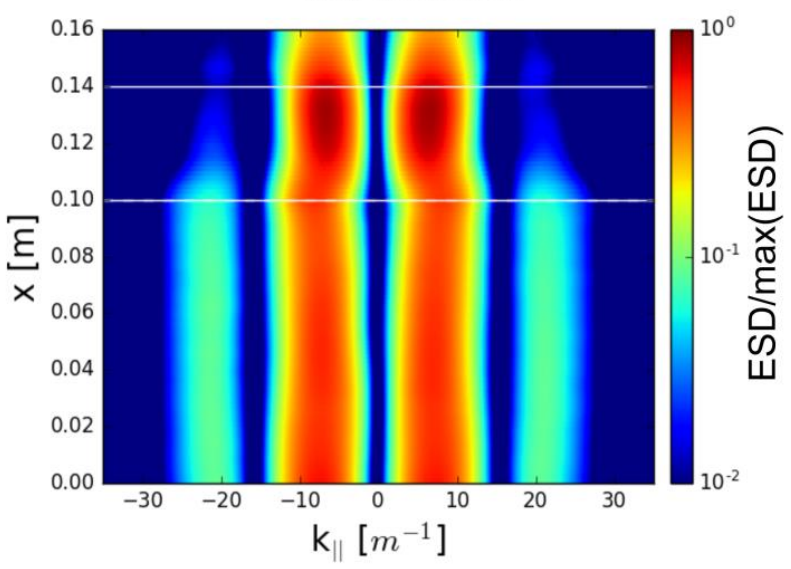

with filament

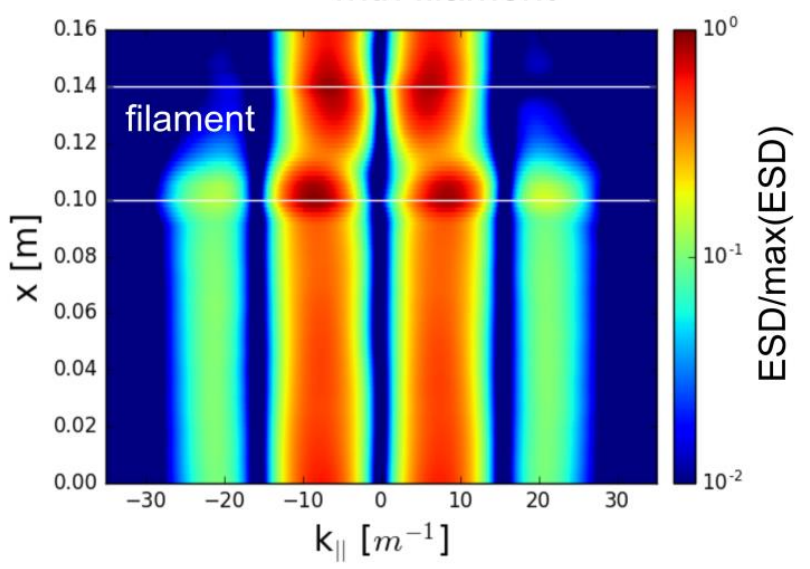

(b) ESD at different $x$ positions
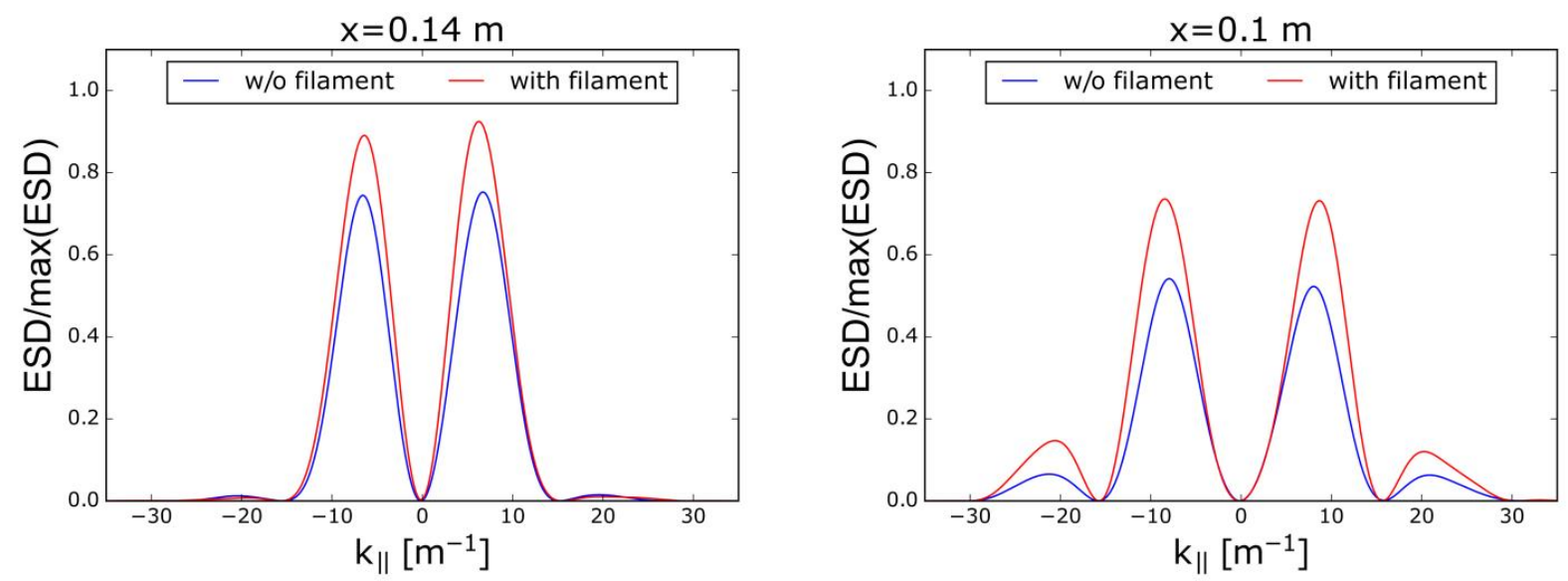

Figure 12. (a) Normalized ESD in the $x$ direction without and with filament. The ESD is normalized to the largest value of the two cases. The horizontal white lines depict the locations of the filament. (b) Normalized ESD at locations of $\mathrm{x}=0.14 \mathrm{~m}$ and $\mathrm{x}=0.10 \mathrm{~m}$. 
(a) $P_{z}$

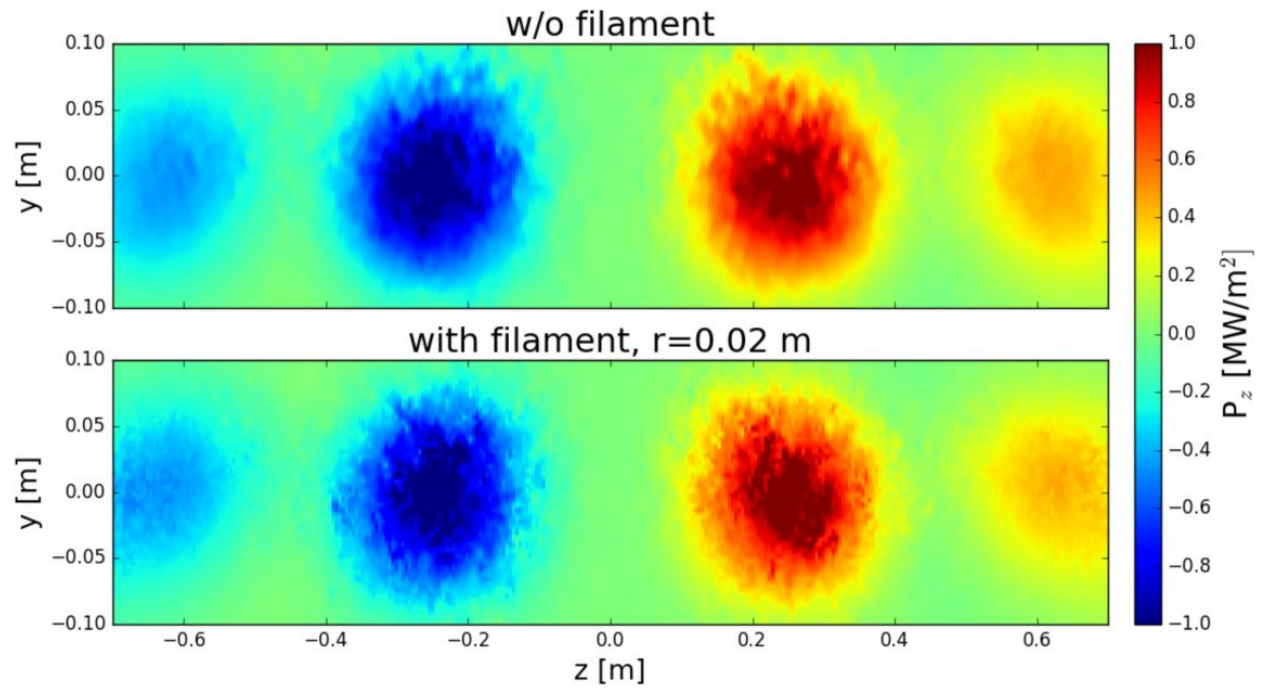

(b) $P_{x}$

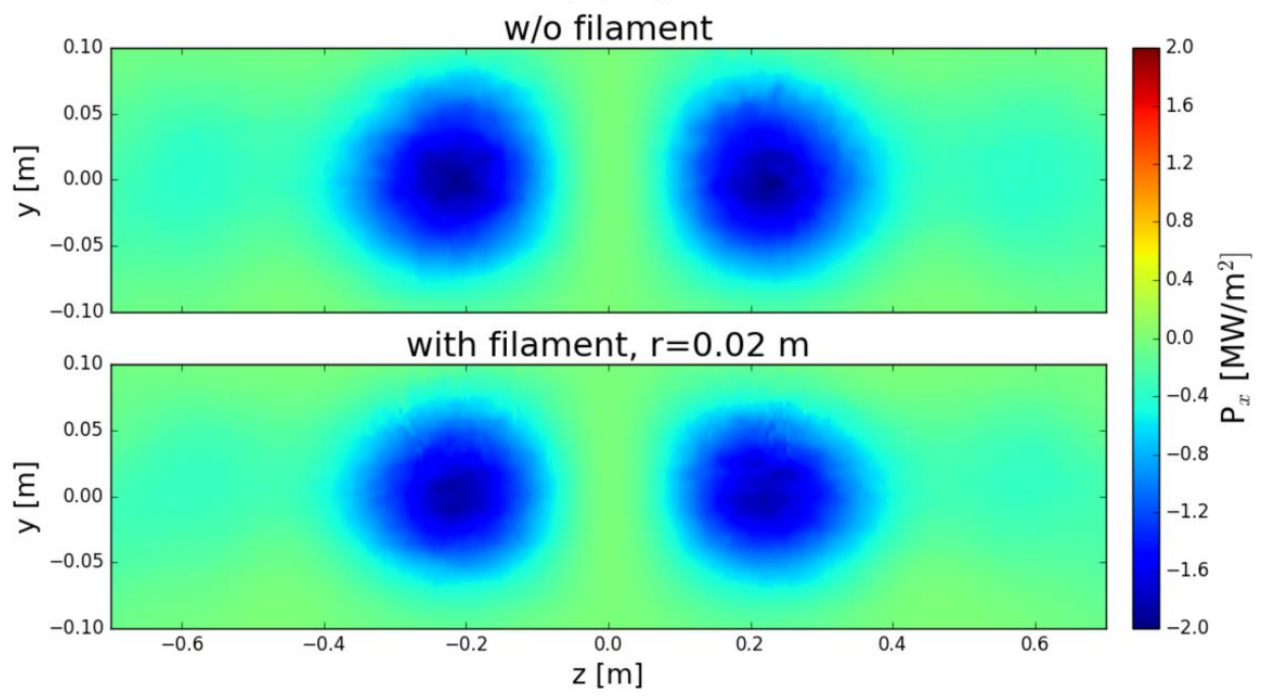

Figure 13. The $z$ and $x$ component of the time averaged Poynting flux in the $y-z$ plane $(x=-0.2 \mathrm{~m})$ for the cases without and with a filament. The filament has a radius of $0.02 \mathrm{~m}$ and a density ratio of $n_{e, F} / n_{e, B}=2$.

In the calculations with an experimental density profile, the power flux to the core is influenced in a small level by the redirection of power flow mechanism. This is shown in Fig. 13, in which the time averaged $P_{z}$ and $P_{x}$ in the $y-z$ plane (at $x=-0.2 \mathrm{~m}$ ) are shown. In the presence of filament, the integration of $P_{x}$ in this $y-z$ plane decreases from $0.130 \mathrm{MW}$ to $0.113 \mathrm{MW}$, suggesting that more power is redirected by the filament into the poloidal and toroidal direction while the total power reaching the core is decreased by $\sim 13 \%$.

\subsection{Resistive losses on the limiter}

As the RF power flow is redirected in the parallel/toroidal direction along the filament, a natural question is whether the power load on the nearby wall structures, especially on the antenna limiters, is increased to a large 
level $\left(10^{6} \mathrm{~W} / \mathrm{m}^{2}\right)$. As most of the RF waves are reflected on the wall, the RF power redirected to the wall is not directly deposited on the wall. The increase of perturbed wave fields near the limiters can however cause induced currents, which can then greatly increase the resistive losses on the limiter surfaces. In the simulations, an impedance boundary condition is set on the limiter surface, which has a Tungsten material (conductivity $\sigma=1.894 \mathrm{e} 7 \mathrm{~S} / \mathrm{m}$ ). The resistive losses per unit area on the limiter surface is calculated by [23]:

$$
W=1 / 2 \sqrt{\omega \mu / 2 \sigma}\left|H_{T}\right|^{2}
$$

in which $\omega$ is the angular frequency, $\mu$ is the permeability and $H_{T}$ is the tangential component of magnetic field. The calculation results (Fig. 14) show that the resistive losses on the limiter surface are increased from the level of $10^{2} \mathrm{~W} / \mathrm{m}^{2}$ to the level of $10^{3} \mathrm{~W} / \mathrm{m}^{2}$ by the filament. The maximum in the increment of resistive losses does not occur at the poloidal position of the filament. This is due to the poloidal asymmetry of the wave fields caused by the filament. Although the resistive power loss on the limiter is increased by an order of magnitude, it is still negligible compare to the heat flux due to the plasma (in the level of $10^{4} \mathrm{~W} / \mathrm{m}^{2}$ ) and too small to cause any damages to the wall.

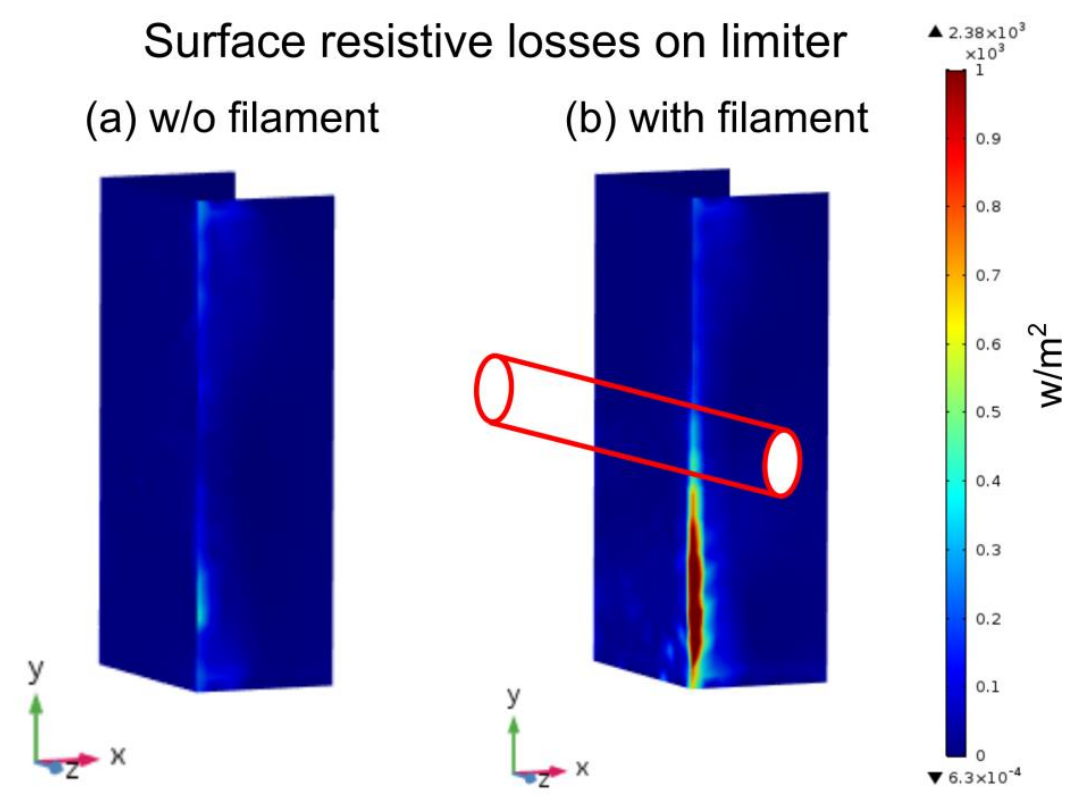

Figure 14. Resistive losses on the limiter surface for the cases without and with a filament.

\section{Conclusions}

Understanding the interaction between filaments and RF waves is important for the improvement of RF heating efficiency. In this paper, the RF power redirection by filaments in the parallel direction (parallel to the magnetic field lines) is studied for the first time. A 3D COMSOL model with a simplified antenna exciting a realistic power spectrum is built. A cylindrical filament, with its density and size comparable to experiments, is assumed to be aligned with the magnetic field lines in the toroidal direction. Cases with constant densities 
representative of particular plasma scenarios and experimental density profiles are considered. Electric fields, Poynting fluxes and power spectrum density are compared for the cases with and without filament.

The results indicate that the power of the fast wave can be redirected by filaments from the radial direction to the parallel direction, with the sacrifice of less power flowing in the radial direction. It is suggested that the increased magnitude of the perpendicular electric and magnetic fields in the filament is the reason for the increased parallel power redirection. The increase of the wave fields subsequently leads to an increase of power spectrum density in the filament.

For the particular cases with constant densities both in the background and filament, the power redirection effect can be very significant, accompanied with the increase of the dominant wavenumbers and the generation of new wavenumbers. Parameter scans with a constant background density of $1 \mathrm{e} 19 \mathrm{~m}^{-3}$ shows that for a fix filament density, the power redirection effects are most significant if the filament size is $\sim 1.75 \mathrm{~cm}$; for a fixed filament size, the parallel redirection of RF power increases nonlinearly as the density ratio between the filament and background increases. For the case with an experimental density profile, the parallel redirection of the RF power is limited toroidally to the antenna vicinity. No new wavenumber is developed in the computed power density spectra, compared to the reference run without filament.

Although the redirected power density in the filament can be as large as $1 \mathrm{MW} / \mathrm{m}^{2}$, this amount of power cannot be directly deposited on the nearby wall structures because of wave reflections on the wall. Instead, because of the induced current, the resistive losses on the antenna limiters can be enhanced by an order of magnitude, to a level $\sim 10^{3} \mathrm{~W} / \mathrm{m}^{2}$. Nevertheless, this amount of power density is still too low to cause any damages to the wall. However, mode-conversion of fast wave to slow wave [5] can happen and be especially strong for certain values of the filament and background density. The mode-converted slow wave can travel along the filament until reaching the wall. Consequently, the sheath can be rectified by the electric fields of the slow wave. The sheath enhanced potential may be a potential damage source to the wall. Further theoretical and experimental work to understand this mechanism is planned in the near future. Experimentally, we plan to use B-dot probes at different toroidal locations of the machine to measure the wave fields of the fast wave and slow wave, and use the reflectometry embedded in the antenna to measure the filament density. Numerically, the extra power losses through sheath rectifications near filaments can be calculated once a 3D sheath boundary condition [24] is included in the model.

Acknowledgments: This work has been carried out within the framework of the EUROfusion Consortium and has received funding from the Euratom research and training programme 2014-2018 and 2019-2020 under grant agreement No 633053. The views and opinions expressed herein do not necessarily reflect those of the European Commission. 


\section{References}

[1] D'Ippolito D. A. et al 2011 Physics of Plasmas 18060501

[2] Stix T. H. 1992 Waves in Plasmas American Inst. of Physics

[3] Ram A. K. et al 2013 Physics of Plasmas 20056110

[4] Ram A. K. et al 2016 Physics of Plasmas 23022504

[5] Myra J. R. et al 2010 Physics of Plasmas 17102510

[6] loannidis Z. C. et al 2017 Physics of Plasmas 24102115

[7] Snicker A. et al 2018 Nuclear Fusion 58016002

[8] Myra J. R. 2014 Physics of Plasmas 21022507

[9] Jacquet P. et al 2011 Nuclear Fusion 51103018

[10] Corre Y. et al 2012 Nuclear Fusion 52103010

[11] Butler H. S. et al 1963 Physics of Fluids 6 1346-55

[12] COMSOL Multiphysics ${ }^{\circledR}$ v. 5.3. www.comsol.com. COMSOL AB, Stockholm, Sweden.

[13] Jacquot J. et al 2013 Plasma Physics and Controlled Fusion 55115004

[14] Tierens W. et al 2019 Nuclear Fusion 59046001

[15] Bobkov V. et al 2013 Nuclear Fusion 53093018

[16] Swanson D. G. 2003 Plasma Waves, 2nd Edition San Diego Academic Press, Inc.

[17] Myra J. R. et al 1994 Physics of Plasmas 1 2890-900

[18] Petty C. C. et al 1999 Nuclear Fusion 39 1421-32

[19] Birkenmeier G. et al 2014 Plasma Physics and Controlled Fusion 56075019

[20] Carralero D. et al 2018 Nuclear Fusion 58

[21] Carralero D. et al 2014 Nuclear Fusion 54123005

[22] Terry J. L. et al 2003 Physics of Plasmas 10 1739-47

[23] Holland S. A. et al 1992 leee Transactions on Magnetics 28 1355-8

[24] Tierens W. et al 2019 Physics of Plasmas 26083501 